\title{
Impact of changing medical workforce demographics in renal medicine over 7 years: Analysis of GMC national trainee survey data
}

\author{
Authors: Matthew PM Graham-Brown, ${ }^{A}$ Hannah K Beckwith, ${ }^{B}$ Simon O'Hare, ${ }^{C}$ Dominic Trewartha, ${ }^{D}$ \\ Aine Burns ${ }^{\mathrm{E}}$ and Sue Carr ${ }^{\mathrm{F}}$
}

\begin{abstract}
Increasing numbers of doctors in training are taking career breaks, with burnout cited as a potential cause. This study analysed General Medical Council (GMC) national training survey data (renal medicine) to understand the impacts of changing workforce demographics on trainee outcomes and wellbeing. Increasing proportions of female, Black, Asian and minority ethnic (BAME), and international medical graduates are entering the workforce. Specialty exam pass rates have fallen and are lower for BAME and international medical graduates in renal medicine. Time to complete higher specialty training has increased for female trainees. Selfreported burnout rates for renal trainees were higher than other medical specialties and highest for male BAME trainees. Burnout was only partially mitigated by less-than-full-time working, but had no impact on progression, sick-leave or time out of training. It is important to recognise changes to the workforce and proactively plan to effectively support a more diverse group of trainees, to enable them to succeed and reduce differential attainment.
\end{abstract}

KEYWORDS: medical education, ethnicity, training, workforce, physician

DOI: $10.7861 /$ clinmed.2020-1065

\section{Introduction}

The UK medical workforce is under pressure. As demand for healthcare services increases, these pressures are amplified and

Authors: ${ }^{\mathrm{A} N I H R}$ academic clinical lecturer and honorary consultant nephrologist, University of Leicester, Leicester, UK, Glenfield Hospital, Leicester, UK, and University Hospitals of Leicester NHS Trust, Leicester, UK; ${ }^{B}$ specialist registrar in renal medicine, Imperial

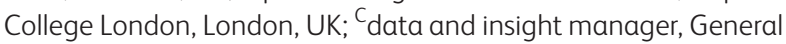
Medical Council, London, UK; ' senior data and insight analyst, General Medical Council, London, UK; ${ }^{\text {p }}$ rofessor of postgraduate medical education and nephrology, University College London, London, UK and Royal Free Hospital, London, UK; F deputy medical director, University Hospitals of Leicester NHS Trust, Leicester, UK and General Medical Council, London, UK without increased medical workforce recruitment, pressure will mount. Increasing numbers of doctors in training are taking career breaks and the 2019 General Medical Council (GMC) state of medical education and practice report showed burnout remains a problem for UK medical trainees. ${ }^{1}$ The report highlighted that the UK is increasingly reliant on recruitment of international medical graduates (IMGs) to maintain trainee workforce, but subsequent attrition of these doctors is substantial. ${ }^{1}$

Studies show that IMGs and UK graduates from Black, Asian and minority ethnic (BAME) backgrounds are more likely to have difficulty forming supportive relationships with trainers and senior clinicians are less likely to have peer support networks and are more likely to suffer the ill effects of burnout, than White UK medical graduates. ${ }^{2}$ This is likely to compound issues related to trainee wellbeing and retention. The importance of supporting trainee wellbeing has been written about widely and a variety of individual and systems level solutions have been trialled with variable successes. ${ }^{3,4}$ Less-than-full-time training (LTFT) has been advocated as a way of ensuring trainees are able to strike a better work-life balance, but while two-thirds of UK medical trainees would consider working LTFT, only around $15 \%$ currently do. ${ }^{5}$ Moreover, the influence of LTFT on mitigating trainee burnout is not known, and neither is the impact on the wellbeing of trainees who continue to work full time.

There have been significant challenges in recruitment to higher specialty training (HST) in several medical specialties over the last decade. Recruitment to renal training reflects many of these challenges, with a reduction in the absolute number of training posts and lower competition ratios for HST over the last 10 years. Renal doctors are responsible for the coordination and delivery of care to patients with kidney diseases, with many contributing to general internal medicine rotas but, despite increasing demand, there is a global shortage of specialists in renal medicine. ${ }^{6,7}$ The UK has one of the lowest numbers of renal specialists per capita compared with similar, publicly-funded healthcare systems and, consequently, many renal centres have already developed extensive, multi-professional teams with extended roles to mitigate medical recruitment challenges. ${ }^{8}$ The causes for recruitment challenges to HST in renal medicine have been attributed to the intensity of the workload, fewer options for private-practice work, work-life balance and high levels of burnout. ${ }^{6,9,10}$ As such, renal trainees serve as a representative medical specialty to explore the impact of changing workforce 
demographics and working patterns on trainee outcomes and satisfaction.

In this study, we performed an in-depth analysis of GMC national training survey (NTS) data over 7 years using renal medicine as a representative medical specialty to gain a granular understanding of the impacts of changing workforce demographic factors (including gender, ethnicity and country of primary medical qualification (PMQ)) on trainee outcomes and wellbeing.

\section{Methods}

We analysed data from the annual GMC NTS from 2012 to 2019. The NTS monitors the quality of postgraduate medical education and training in the UK and is circulated to over 45,000 doctors in training and 40,000 trainers across the four UK nations. The response rate is usually above $90 \%$ for trainees and $40 \%$ for trainers. GMC NTS data were collected prospectively and analysed anonymously. We analysed data extracted from the survey each year for doctors in HST in the 30 medical specialties and three sub-specialties. ${ }^{11,12}$ Data were extracted regarding gender, ethnicity, country of PMQ, working pattern (LTFT or full time) and self-reported burnout.

\section{Changes in trainee characteristics recruited to ST3 and} working practices from 2012 to 2019

Ethnicity, country of PMQ and gender were compared for all trainees who completed the GMC NTS between 2012 and 2019 and compared with the demographics of other medical specialties.

\section{Outcomes for trainees in renal medicine}

Annual review of competence progression (ARCP) outcomes (favourable and unfavourable) and specialty exam (SCE) pass rates (first attempt) were compared for renal trainees and trainees in other medical specialties between 2017 and 2019. Outcomes in the SCE and at ARCP for renal trainees were reviewed based on gender, ethnicity and country of PMQ.

\section{Rates of burnout among renal trainees}

Burnout was measured using the seven work-related questions from the Copenhagen Burnout Inventory. ${ }^{13}$ Questions were scored as a scale from 0-100. Respondents' mean reported scores across all seven questions were categorised into one of three levels of burnout risk: low, moderate or high. Rates of burnout among renal trainees were compared with rates of burnout for trainees in other medical specialties. Differences in severity of burnout for trainees were explored based on gender, ethnicity and country of PMQ.

\section{Statistical testing}

Statistical analysis was undertaken using SPSS 27 software (IBM, Armonk, USA). Parametric data are expressed as mean \pm standard deviation. Non-parametric data are expressed as median (interquartile range (IQR)). Nominal/categorical (ie male/female, pass/fail, satisfactory/unsatisfactory, BAME/White, UK PMQ/IMG) data are expressed as 'count' (\%) and chi-squared tests were used to establish statistical differences between groups. A $p<0.05$ was considered statistically significant. The reported findings include the Pearson chi-squared value and asymptotic significance (two-sided).

\section{Results}

The GMC trainee survey included data from 11,886 participants between 2012 and 2019 (renal medicine trainees $n=627$; trainees in all other medical specialties $n=11,259$ ). Table 1 shows the number and demographic characteristics of respondents to the surveys for each year between 2012 and 2019.

\section{Changes in renal trainee characteristics and working patterns from 2012 to 2019}

Fig 1 shows the demographics of the renal trainees entering HST (ST3) from 2012-2019. The proportion of women entering HST and the proportion of trainees from BAME backgrounds and those with a non-UK PMQ has increased. There has been an increase in the number of female doctors on the specialist register for renal medicine between 2014 and 2019 (one in three on the specialist register in 2019 compared with one in four in 2014; supplementary material S1, Fig S1), with an increase in the age of doctors completing HST between 2014 and 2019. Median age for women and men joining the specialist register in 2014 were 37.2 years (IQR 10.3) and 36.6 years (IQR 11.8) vs 38.2 years (IQR 11.3) and 37 years (IQR 10.5) for trainees joining the specialist register in

Table 1. The demographics of trainees who completed the General Medical Council national training survey

\section{Renal medicine}

Total, Female, n

201278

$2013 \quad 77$

$2014 \quad 69$

201575

$2016 \quad 67$

201794

201876

201991

\section{All medical specialties}

\begin{tabular}{llllllllll} 
BAME, & $\begin{array}{l}\text { White, } \\
\%\end{array}$ & $\begin{array}{l}\text { IMG, } \\
\%\end{array}$ & $\begin{array}{l}\text { UK Grad, } \\
\%\end{array}$ & $\begin{array}{l}\text { Total, } \\
\mathbf{n}\end{array}$ & $\begin{array}{l}\text { Female, } \\
\%\end{array}$ & $\begin{array}{l}\text { BAME, } \\
\%\end{array}$ & $\begin{array}{l}\text { White, } \\
\%\end{array}$ & $\begin{array}{l}\text { IMG, } \\
\%\end{array}$ & $\begin{array}{l}\text { UK Grad, } \\
\%\end{array}$ \\
41 & 59 & 36 & 64 & 1,436 & 51 & 50 & 50 & 32 & 68 \\
33 & 67 & 26 & 74 & 1,394 & 53 & 43 & 57 & 24 & 76 \\
41 & 59 & 20 & 80 & 1,408 & 54 & 42 & 58 & 25 & 75 \\
43 & 57 & 28 & 72 & 1,410 & 51 & 42 & 58 & 24 & 76 \\
53 & 47 & 28 & 72 & 1,399 & 53 & 41 & 59 & 23 & 77 \\
45 & 55 & 21 & 79 & 1,391 & 52 & 43 & 57 & 25 & 75 \\
63 & 38 & 29 & 71 & 1,330 & 52 & 45 & 55 & 25 & 75 \\
53 & 47 & 37 & 63 & 1,491 & 52 & 47 & 53 & 28 & 72 \\
\hline
\end{tabular}

BAME = Black, Asian and minority ethnic; Grad = graduate; $I M G$ = international medical graduate . 


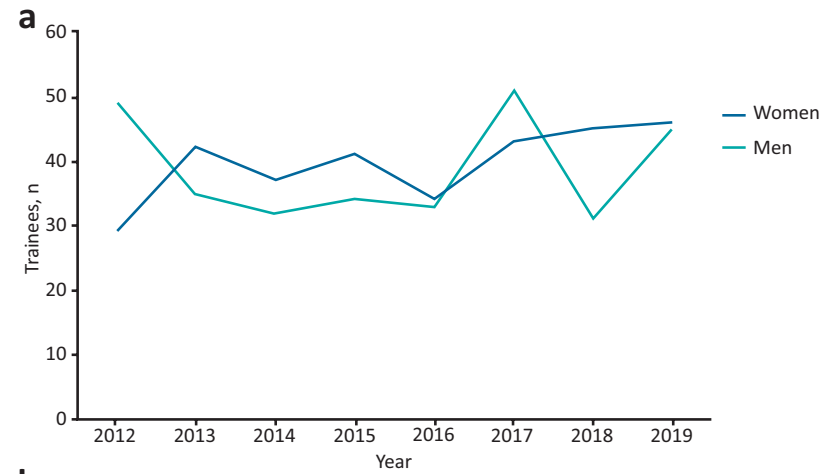

b
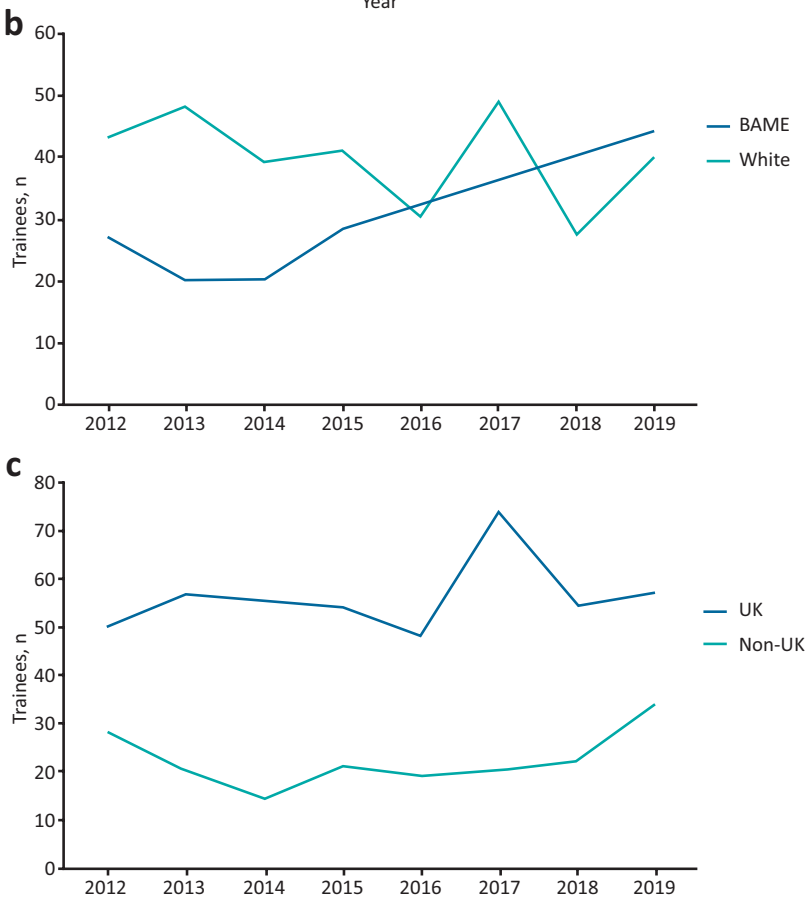

Fig 1. Changes in the renal trainee entering renal training at ST3 between 2012 and 2019. a) Gender. b) Ethnicity. c) Country of primary medical qualification graduation. BAME $=$ Black, Asian and minority ethnic; ST3 = specialty trainee year 3 .

2019. This is despite no increase in the age at which these doctors gained their PMQ.

\section{Outcomes for renal trainees}

\section{SCES}

Between 2017 and 2019, renal trainees ( $n=161)$ had the lowest success rate at their SCE at the first attempt compared with trainees from other medical specialties (Table 2). For renal trainees, there were no differences between SCE pass rates between men $(n=83)$ and women ( $n=78 ; 81.9 \%$ vs $79.5 \%$; chi-squared 0.154 $(1 ; n=161) ; p>0.5)$. White doctors $(n=80)$ were more likely to pass the exam first time than BAME doctors ( $n=76 ; 88.8 \%$ vs $75.0 \%$; chi-squared 5.003 (1; $n=156) ; p<0.01)$, as were doctors with a UK PMQ ( $n=124$ ) vs IMG doctors ( $n=37 ; 86.3 \%$ vs $62.2 \%$; chi-squared 10.671 ( $1 ; n=161) ; p<0.001)$. There was no statistically significant difference in pass rate between White doctors $(90.8 \%)$ than BAME doctors with a UK PMQ (85.1\%).
ARCPS

Between 2017 and 2019, renal trainees ( $n=941$ ) outcomes at ARCP were comparable with other medical specialties (Table 3). Male renal trainees $(n=344)$ were more likely to have an unsatisfactory ARCP outcome than female trainees ( $n=379 ; 4.4 \%$ vs $0.8 \%$; chi-squared $9.46(1 ; n=723) ; p<0.01)$. BAME renal trainees $(n=399)$ were significantly more likely to receive an unsatisfactory outcome at ARCP than White trainees ( $n=502 ; 5.3 \%$ vs $2.4 \%$; chi-squared 5.199 ( $1 ; n=901) ; p<0.05)$. There was no difference between ARCP outcomes for BAME $(n=234)$ or White $(n=460)$ renal trainees whose PMQ was in the UK $(2.4 \%$ vs $3.0 \%$, chi-squared $1.86(1 ; n=207) ; p>0.10)$ or BAME $(n=165)$ or White $(n=42)$ IMG trainees ( $8.5 \%$ vs $2.4 \%$; chi-squared $0.22(1 ; n=694)$; $p>0.50)$.

\section{Completion of training}

Time taken from PMQ to completion of HST increased for female renal trainees between 2014 and 2019, but not men (Table 4). The comparative outcomes for renal and other medical trainees are shown in Fig 2. One-third of renal trainees who started HST in 2012 were still in renal training in 2019; higher than in other medical specialties. For renal trainees who had left training, the majority had left UK practice and had no designated body information. Thirty per cent remained in UK medical practice but not in training, and $10 \%$ had re-entered a different UK training programme. Attrition from renal training was higher than in other medical specialties.

\section{Self-reported rates of burnout among renal trainees}

In the 2019 GMC trainee survey, self-reported rates of burnout were higher among renal trainees compared with trainees in other medical specialties (Fig 3) and higher than most other medical specialties (supplementary material S1, Fig S2). Differences were particularly pronounced among male renal trainees who reported higher levels of burnout than female colleagues and male colleagues in other medical specialties. Among renal trainees, rates of burnout were higher for BAME trainees compared with White trainees, and this pattern was more pronounced than for other medical specialties. There were higher rates of burnout reported among IMG renal trainees, the opposite to other medical specialties. Overall self-reported rates of burnout between full-time and LTFT renal trainees were similar, although full-time trainees reported increased 'high' levels of burnout. For other medical specialties, LTFT trainees consistently reported lower rates of burnout compared with full-time colleagues.

Burnout did not seem to influence the likelihood of trainees progressing in training or need for sick leave or time out of training in the subsequent year.

\section{Discussion}

This analysis demonstrates important changes in the demographics of renal trainees over the last 7 years. There are increasing proportions of female trainees, trainees from BAME backgrounds and IMGs. Burnout is higher for renal trainees than for other medical specialties, highest for male BAME trainees and not mitigated by LTFT training as in other medical specialties.

\section{Implications of changing workforce demographics}

These changes in workforce demographics have implications for workforce planning. The proportion of female renal trainees has 
Table 2. Success rates for candidates siting specialty exams between 2017 and 2019

\begin{tabular}{lllll} 
Specialty & $\begin{array}{l}\text { Number of } \\
\text { candidates, }\end{array}$ & $\begin{array}{l}\text { Number of } \\
\text { attempts, }\end{array}$ & $\begin{array}{l}\text { Number of successful } \\
\text { attempts, }\end{array}$ & $\begin{array}{l}\text { Pass rate for } \\
\text { all attempts, } \\
\%\end{array}$ \\
Medical oncology & $\mathbf{n}$ & $\mathbf{n}$ & $\mathbf{n}$ & 94.6 \\
Geriatric medicine & 111 & 111 & 105 & 94.2 \\
Dermatology & 412 & 412 & 388 & 91.7 \\
Infectious diseases & 157 & 157 & 144 & 89.3 \\
Neurology & 75 & 75 & 67 & 86.8 \\
Acute medicine & 174 & 174 & 151 & 86.6 \\
Pan-European gastroenterology and hepatology (ESEGH) & 246 & 187 & 162 & 86.6 \\
Endocrinology and diabetes & 187 & 246 & 213 & 86.0 \\
Palliative medicine & 229 & 229 & 197 & 85.4 \\
Gastroenterology & 123 & 123 & 105 & 85.0 \\
Rheumatology & 113 & 113 & 96 & 84.1 \\
Respiratory medicine & 145 & 145 & 122 & 81.6 \\
Nephrology & 310 & 310 & 253 & 80.7 \\
All SCE & 161 & 161 & 130 & 87.3 \\
\hline SCE = specialty exams. & 2,443 & 2,443 & 2,133 & \\
& & &
\end{tabular}

increased from $37 \%$ in 2012 to $51 \%$ in 2019 . Female renal trainees are also taking longer to complete training compared with 5 years ago, with a median increase in training time of 1 year between 2014 and 2019. It is not clear why this increased time in training has occurred but it may be related to an increase in career breaks, perhaps as parental leave, increased time out of programme or because of an increase in LTFT working. Whatever the reason, renal workforce planning must take account of these changes as female renal specialists now are older than 5 years ago and may have chosen a different working pattern to the conventional full-time contract. These changes have implications for service delivery at consultant level, especially considering the relative under-representation of specialist renal doctors in the UK medical workforce compared with international numbers. ${ }^{8}$

The increased proportion of renal trainees from BAME backgrounds and IMG trainees has implications for trainers, training programmes and workforce planning. It has been shown that BAME and IMG doctors have more difficulty forming supportive relationships with supervisors and senior clinicians than White UK graduates. ${ }^{2}$ In addition, the GMC Fair to refer? report highlighted that BAME and IMG doctors are more likely to be referred to their regulator than White doctors. The authors identified that a lack of supportive induction and constructive feedback adversely impacted some doctors' ability to learn from their mistakes and improve their performance. ${ }^{13}$ The report highlights the importance of appropriate induction and building time for BAME and IMG trainees to form supportive relationships with supervisors and trainers. Our analysis identified a discrepancy in pass rates in the SCE for renal trainees from a BAME background and IMGs compared with White UK-trained trainees, with country of PMQ having the greatest influence on SCE success rate. These data largely mirrored the ARCP outcomes for renal trainees, although additionally male renal trainees were more likely to have an unsatisfactory ARCP outcome than women. Differential attainment in postgraduate examinations has been identified by the GMC and others as an important issue which must be understood and addressed. ${ }^{14}$ These issues may also create a more stressful working environment and contribute to the higher levels of burnout reported by these groups. Tailored induction and support programmes for IMG doctors may be a way of mitigating these differences in attainment, but will require sound methodological design and evaluation.

\section{Burnout in renal medicine trainees}

Renal trainees consistently reported higher levels of burnout than trainees in other medical specialties. The reasons for these differences are not clear from this analysis. Reports have identified that working in pressurised healthcare environments impacts doctor wellbeing and retention within the NHS. ${ }^{15,16}$ Junior doctors in the UK view renal medicine as complex, with a heavy workload which can adversely impact work-life balance. ${ }^{9}$ Similarly, in the USA, emotional exhaustion and feelings of depersonalisation were highly prevalent in renal trainees, particularly among women. ${ }^{17}$ High levels of burnout among renal trainees are, therefore, unsurprising. More flexible working patterns have been suggested as a way of improving trainee wellbeing. ${ }^{4,18}$ Increasing numbers of trainees are taking breaks in training, and a lack of flexibility in training programmes is consistently cited as a reason for this. ${ }^{18}$ While improving flexibility in training helps reduce work pressure and burnout it is unlikely to be effective as an isolated solution. Indeed, a randomised trial in the USA assessed the impact of flexible training schedules on the satisfaction of internal medicine trainees. While programme directors were happy with the trialled programme, interns were less happy and rates of burnout were unchanged. ${ }^{19}$ A more comprehensive review of working patterns, environments, training and trainee support is needed to have a meaningful impact on trainee wellbeing and reduce attrition from training. ${ }^{20}$ 
Table 3. Success rates of annual review of competence progression between 2017 and 2019

Specialty

Aviation and space medicine

Medical ophthalmology

Metabolic medicine

Pharmaceutical medicine

Audio vestibular medicine

Clinical neurophysiology

Acute internal medicine

Immunology

Cardiology

Nuclear medicine

Sport and exercise medicine

General (internal) medicine

Rehabilitation medicine

Respiratory medicine

Endocrinology and diabetes mellitus

Geriatric medicine

Gastroenterology

Palliative medicine

Haematology

Genito-urinary medicine

Infectious diseases

Renal medicine

Allergy

Rheumatology

Neurology

Paediatric cardiology

Medical oncology

Clinical genetics

Clinical pharmacology and therapeutics

Dermatology

Stroke medicine

Hepatology

Tropical medicine

All ARCPs

$\mathrm{ARCP}=$ annual review of competence progression.

For trainees in renal medicine, burnout appears to disproportionately affect men from a BAME background. This group make up an increasing proportion of the trainee workforce and these findings should be a particular concern for trainers and training programme directors. The data show that attrition from renal training programmes is already higher than other medical specialties and renal trainees spend a longer

\section{Outcomes (excluding Unsatisfactory outcomes} exam failure only),

\section{n}

5

26

37

341

42

90

910

96

2,263

30

115

6,474

160

1,622

1,080

1,691

1,592

585

1,454

313

620

941

30

752

985

120

723

175

99

690

15

4

4

24,084

\section{(excluding exam failure only)}

n

1

4

5

42

4

8

79

8

176

2

7

379

9

87

53

82

67

23

57

12

23

33

1

24

29

3

17

4

2

9

-

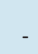

-

1,250
Unsatisfactory outcome rate $\%$

20.0

15.4

13.5

12.3

9.5

8.9

8.7

8.3

7.8

6.7

6.1

5.9

5.6

5.4

4.9

4.8

4.2

3.9

3.9

3.8

3.7

3.5

3.3

3.2

2.9

2.5

2.4

2.3

2.0

1.3

0.0

0.0

0.0

5.2

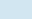

5.6

4

8

4.2

9

9

8

7

5

3

2

9

.3

0

0.0

\section{(excluding exam failure only) \\ exam failure only),} 0 3

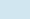

7

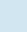

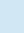

5

time completing training than colleagues in other medical specialties. The high levels of burnout reported by BAME and IMG trainees in renal medicine could further increase attrition from training programmes. These doctors must receive appropriate support from clinical leaders, mentors and sponsors who, in turn, must be representative of a diverse range of backgrounds. 


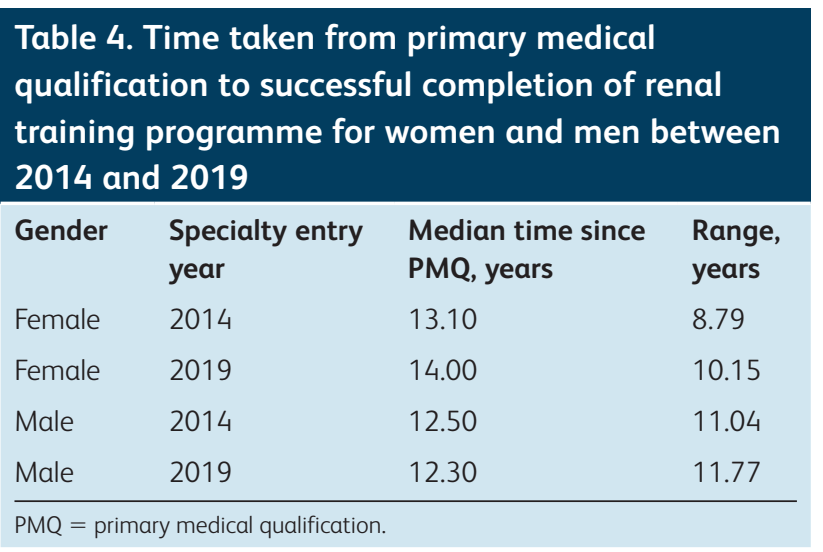

While levels of burnout were lower among LTFT trainees in this analysis, it is unknown whether increases to the proportions of trainees in LTFT training would improve trainee burnout. It will be important to understand the implications of LTFT training on the clinical workloads and service commitments of trainees who work full time. The consequence of an increase in the proportion of LTFT trainees in the workforce should be carefully considered in the design of rotas and training programme placements to ensure that satisfaction and training quality does not adversely affect trainees who work full time.

\section{Possible solutions}

Managing workload and increasing trainee autonomy may improve trainee wellbeing immediately. ${ }^{4} \mathrm{~A}$ revision of the ways in which rotas are staffed is essential, in addition to facilitating attendance at high-value training activities as well as delivering clinical service provision. The specialty specific, operationalisation of such rotas with a 'self-service' element would give trainees more control over daily working activities and allow them to plan and prepare for periods of high-volume clinical service work with the knowledge that a less intense period of high-value training would follow. The Royal College of Physicians chief registrars have been instrumental in leading changes to improve junior and middle-grade rotas and could play a key role in developing and testing the impact of self-service training rotas on wellbeing and burnout. $^{21}$

Longer-term solutions that support the medical workforce to deliver high-quality patient care is key to sustaining recruitment and retention. Ensuring opportunities are equitable for all members of the workforce is a priority and gender, race or other demographic discriminator must not adversely affect career opportunities or development. ${ }^{22,23}$ The recent We are the NHS: People plan for 2020/2021 - action for us all highlights the need to act to improve staff wellbeing, encourage flexible working and create an inclusive culture. ${ }^{24}$ This recognises the importance of investment in the development and regulation of new healthcare roles to support multi-professional teams to deliver patient care. Physician associates, advanced clinical practitioners and assistant practitioners have demonstrated their ability to support delivery of safe, high-quality patient care and, by doing so, trainees have more time to undertake training-related activities. Within renal medicine (and other specialties), a potential sustainable solution to support workforce challenges might include more defined roles and responsibilities for medically associated professionals (MAPs)
Fig 2. Outcomes for trainees who commenced training in 2012. a) Renal trainees. b) Medical trainees (excluding renal trainees). $\mathrm{GP}=$ general practitioner.

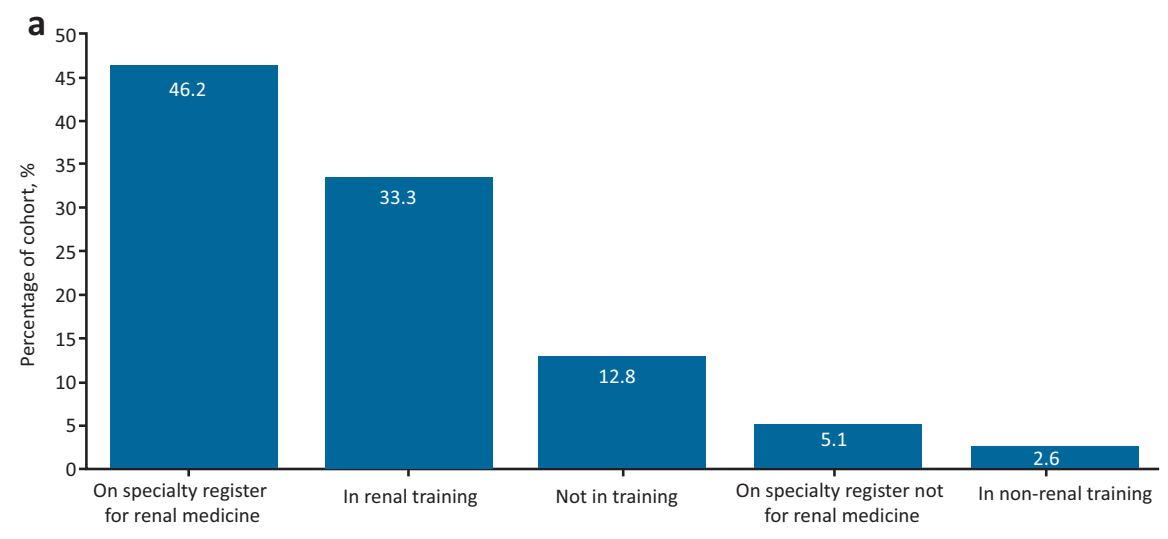

b

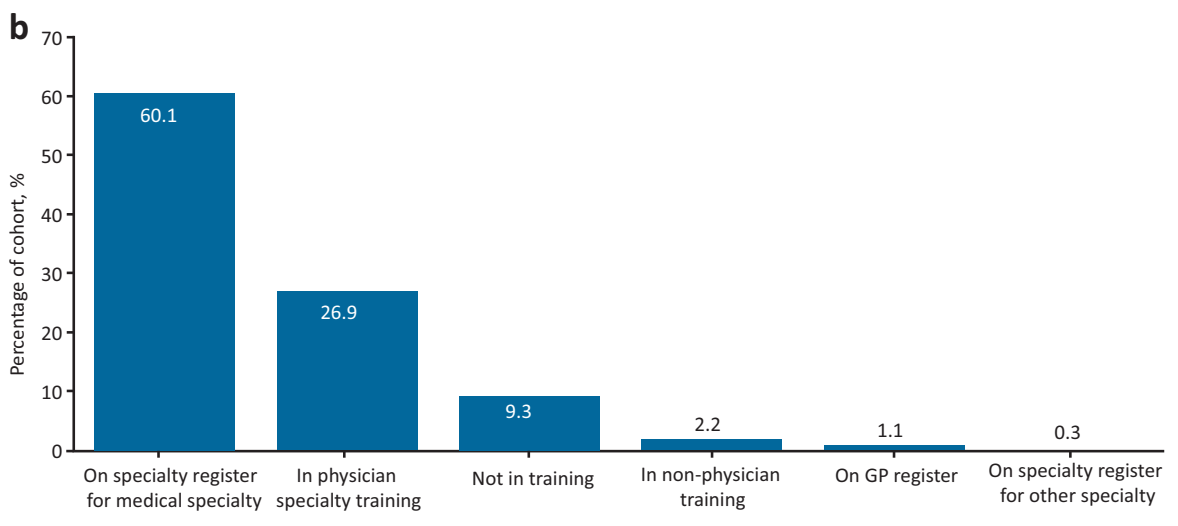



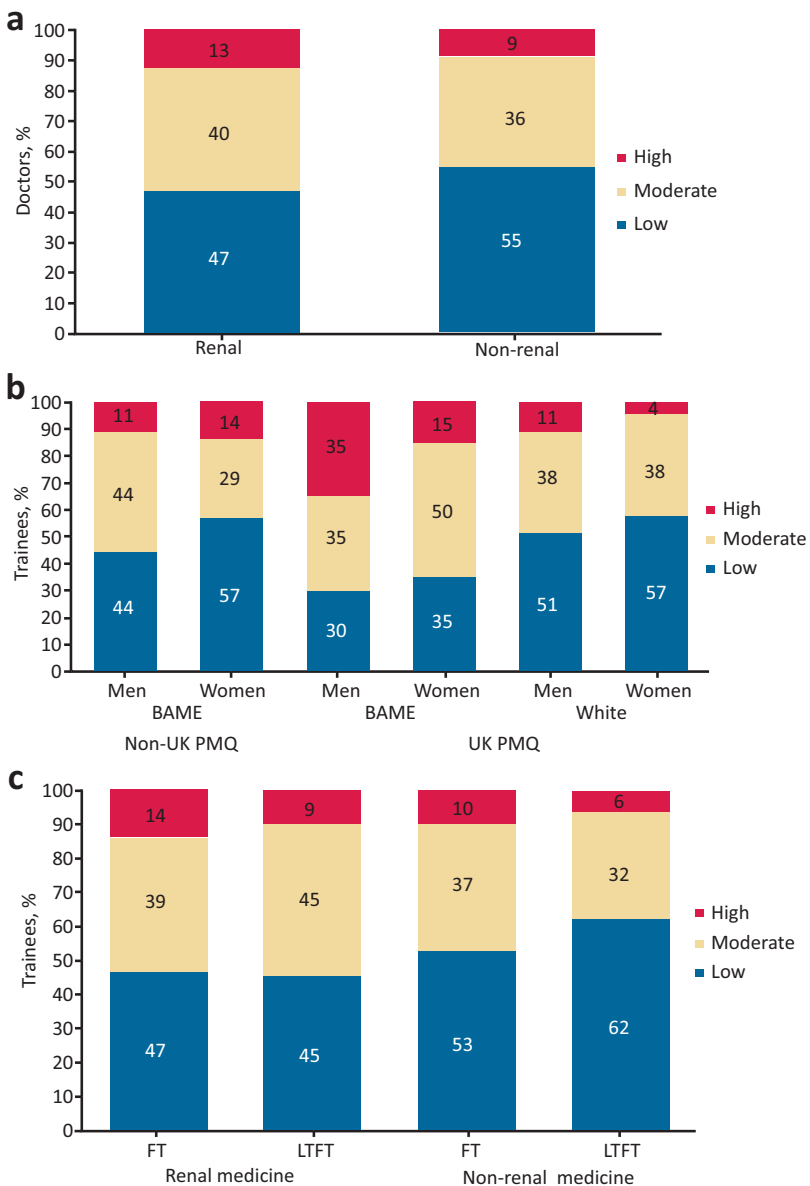

Fig 3. Rates of burnout for renal trainees. a) By specialty. b) By gender, ethnicity and UK vs non-UK primary medical qualification. c) By specialty and full time or less-than-full-time. BAME = Black, Asian and minority ethnic; $\mathrm{FT}=$ full time; $\mathrm{LTFT}=$ less-than-full-time; $\mathrm{PMQ}=$ primary medical qualification.

with extended healthcare roles embedded within clinical service models to support patient care flows and the workload of trainees. Renal services have embraced new ways of working in some areas, but these initiatives are by no means ubiquitous or sustainable; for example, delivery of many aspects of maintenance haemodialysis programmes can be safely managed by experienced allied health professionals in extended roles, but units where services are organised in this way are the exception not the rule. Future regulation of MAPs will enable them to work to their potential and contribute to the development of a safe and flexible multiprofessional workforce which provides high-quality care to patients and supports a work environment that delivers high-quality training for all.

Continuing to attract trainees to renal medicine must be a priority for the specialty. Early exposure to specialty, the flexibility to develop portfolio careers alongside education, management and academia are certainly an imperative, but equally addressing concerns about future financial remuneration also seem important for many. ${ }^{25-27}$ Combining elements from all these solutions are most likely to have an impact on improving training.

\section{Conclusion}

This study describes significant demographic changes in the renal trainee workforce over a 7-year period. The increasing numbers of female trainees, BAME doctors and IMGs has implications for workforce planning. Trainees are taking longer to train, there is increased attrition from programmes and high rates of burnout for renal trainees compared with other medical specialties which disproportionately affects BAME and IMG trainees. It is important to recognise the changes in the trainee workforce and proactively plan to effectively support a more diverse group of trainees with high-quality induction and supervision to enable them to succeed and reduce differential attainment. Continuing to transform training to support trainee retention and to develop and embed a multi-professional healthcare workforce that delivers high-quality patient care and to support doctors in training is a priority.

\section{Summary}

\section{What is known?}

The pressures on modern medical training has led to significant attrition from training programmes for trainees in renal medicine.

\section{What is the question?}

To understand the changing demographics of the renal workforce compared with other medical workforce through analysis of General Medical Council national training survey data from 2012 to 2019. To gain an understanding of the different rates of burnout between renal trainees and other medical trainees and between trainees of different demographic backgrounds. To assess differences in outcomes of renal trainees at annual review of competence progression (ARCP) and in the specialty exams based on different demographic factors.

\section{What was found?}

We found increasing proportions of female, Black, Asian and minority ethnic (BAME) and international medical graduates are entering the renal trainee workforce. Self-reported burnout rates for renal trainees were higher than other medical specialties and highest for male BAME trainees. Renal trainees from BAME backgrounds and international medical graduates are less likely to pass the specialty exam at the first attempt and more likely to have an unsatisfactory ARCP outcome.

\section{What is the implication for practice now?}

Recognising and understanding the changes to the trainee workforce allows proactive planning to effectively support a more diverse group of trainees and reduce differential attainment.

\section{Supplementary material}

Additional supplementary material may be found in the online version of this article at www.rcpjournals.org/clinmedicine: S1 - Supplementary figures.

\section{Conflicts of interest}

Sue Carr is deputy medical director of the GMC. Simon O'Hare and Dominic Trewartha are GMC employees.

\section{References}

1 General Medical Council. The state of medical education and practice in the UK, 2019. GMC, 2019. 
2 Woolf K, Rich A, Viney R, Needleman S, Griffin A. Perceived causes of differential attainment in UK postgraduate medical training: a national qualitative study. BMJ Open 2016;6:e013429.

3 Ripp JA, Privitera MR, West CP et al. Well-being in graduate medical education: a call for action. Academic Medicine 2017;92:914-7.

4 West M, Coia D. Caring for doctors, caring for patients. General Medical Council, 2019.

5 Rimmer A. Allowing more doctors to work less than full time could reduce burnout, says royal college. BMJ 2019;366:15778.

6 Sharif MU, Elsayed ME, Stack AG. The global nephrology workforce: emerging threats and potential solutions! Clinical Kidney Journal 2016;9:11-22.

7 Osman MA, Alrukhaimi M, Ashuntantang GE et al. Global nephrology workforce: gaps and opportunities toward a sustainable kidney care system. Kidney International Supplements 2018;8:52-63.

8 Bello AK, Levin A, Manns BJ et al. Effective CKD care in European countries: challenges and opportunities for health policy. American Journal of Kidney Diseases 2015;65:15-25.

9 Karangizi AH, Chanouzas D, Mahdi A, Foggensteiner L. How can we make renal medicine careers more appealing to UK trainees? Clinical Kidney Journal 2019;12:756-9.

10 Pawłowicz E, Nowicki M. Burnout syndrome among nephrologistsa burning issue-results of the countrywide survey by the Polish Society of Nephrology. BMC Nephrol 2020;21:177.

11 Joint Royal Colleges of Physicians Training Board. Specialties. JRCPTB, 2020. www.jrcptb.org.uk/specialties.

12 General Medical Council. GMC approved post-graduate curricula. GMC, 2020. www.gmc-uk.org/education/standards-guidance-andcurricula/curricula\#approved_curricula

13 General Medical Council. Fair to refer?: Reducing disproportionality in fitness to practise concerns reported to the GMC. GMC, 2019.

14 General Medical Council. Differential attainment. GMC, 2020. www.gmc-uk.org/education/standards-guidance-and-curricula/ projects/differential-attainment

15 Simmer S, Raleigh V. The risks to care quality and staff wellbeing of an NHS system under pressure. Oxford: Picker, 2018.
16 West M. The NHS crisis of caring for staff: what do we need to do? The King's Fund, 2019. www.kingsfund.org.uk/blog/2019/03/nhscrisis-caring

17 Agrawal V, Plantinga L, Abdel-Kader K et al. Burnout and emotional well-being among nephrology fellows: a national online survey. J Am Soc Nephrol 2020;31:675-85.

18 Academy of Medical Royal Colleges. Guidance for flexibility in postgraduate training and changing specialties. AoMRC, 2020.

19 Desai SV, Asch DA, Bellini LM et al. Education outcomes in a dutyhour flexibility trial in internal medicine. N Engl ] Med 2018;378: 1494-508.

20 British Medical Association. Caring, supportive, collaborative: a future vision for the NHS. BMA, 2020.

21 Exworthy M, Snelling I. Evaluation of the RCP's chief registrar programme. Royal College of Physicians, 2017.

22 O'Lone E, Webster AC. Barriers to the professional advancement of women in nephrology. Clin J Am Soc Nephrol 2019;14:1399-401.

23 Woolf K. Differential attainment in medical education and training. BMJ 2020:368:m339.

24 NHS England, NHS Improvement, Health Education England. We are the NHS: People plan for 2020/2021 - action for us all. NHS, 2020.

25 Beckwith H, Kingsbury M, Horsburgh J. Why do people choose nephrology? Identifying positive motivators to aid recruitment and retention. Clinical Kidney Journal 2018;11:599-604.

26 Lane CA, Healy C, Ho M, Pearson S, Brown MA. How to attract a nephrology trainee: quantitative questionnaire results. Nephrology 2008;13:116-23.

27 Huilgol SG. Nephrology workforce in India - a fellow's perspective. The Open Urology \&\#38; Nephrology Journal 2015;8:64-5.

Address for correspondence: Prof Sue Carr, General Medical Council, Regent's Place, 350 Euston Road, London NW1 3JN, UK. Email: sue.carr1@gmc-uk.org

Twitter: @suecarr4360; @drmattgb; @hannahksb 